\title{
A New Special Function and Its Application in Probability
}

\author{
Zeraoulia Rafik (D), ${ }^{1}$ Alvaro H. Salas, ${ }^{2}$ and David L. Ocampo ${ }^{2,3}$ \\ ${ }^{1}$ University Batna, Algeria \\ ${ }^{2}$ Universidad Nacional de Colombia, Colombia \\ ${ }^{3}$ Universidad de Caldas-Colombia, Colombia \\ Correspondence should be addressed to Zeraoulia Rafik; zeraouliarafik@gmail.com
}

Received 28 June 2018; Revised 24 August 2018; Accepted 17 September 2018; Published 1 November 2018

Academic Editor: A. Zayed

Copyright (C) 2018 Zeraoulia Rafik et al. This is an open access article distributed under the Creative Commons Attribution License, which permits unrestricted use, distribution, and reproduction in any medium, provided the original work is properly cited.

\begin{abstract}
In this note we present a new special function that behaves like the error function and we provide an approximated accurate closed form for its CDF in terms of both Chèbyshev polynomials of the first kind and the error function. Also we provide its series representation using Padé approximant. We show a convincing numerical evidence about an accuracy of $10^{-6}$ for the approximants in the sense of the quadratic mean norm. A similar approach may be applied to other probability distributions, for example, Maxwell-Boltzmann distribution and normal distribution, such that we show its application using both of those distributions.
\end{abstract}

\section{Introduction}

Integrals of the error function, see $\left({ }^{1}\right)$, occur in a great variety of applications usually in problems involving multiple integration where the integrand contains exponentials of the squares of the argument; an example of applications can be cited from atomic physics astrophysics and statistical analysis. It comes into our mind to seek for the integration of such functions $f(x)$ power its antiderivative $g(x)$. We have got example (1) where it is the power of two distributions related to normal distribution [1] as shown below such that $f(x)=$ $e^{-x^{2}}$ and $g(x)=\operatorname{erf}(x)$

$$
I(a)=\int_{0}^{a}\left(e^{-x^{2}}\right)^{\operatorname{erf}(x)} d x
$$

with $\operatorname{erf}(x)$ is called error function and it is defined in (24).

$$
\frac{2}{\sqrt{\pi}} \int_{0}^{x} e^{-t^{2}} d t=\operatorname{erf}(x)
$$

1.1. Numerical Approximation of $\int_{0}^{a}\left(e^{-x^{2}}\right)^{\operatorname{erf}(x)} d x$ in Some Ranges Values. Now, if we really need a simple expression for $I(a)$ in some range of values, there are ways to get various approximations.

The function is very nice. It goes to its limit at $\infty$ very fast. Figure 1 shows the plot of $I(a)$ for $a \in[0,10]$.

Therefore (depending on the accuracy we need) we can easily take $I(a)=I(\infty)$ for $a>a_{0}$ with $a_{0}$ around 3 or 4 .

Mathematica gives the following for the first 100 digits.

$$
\begin{aligned}
& I(\infty) \\
& =0.9721069927691785931510778754423911755542721833855699009722910408441888759958220033410678218401258734
\end{aligned}
$$

Now, what can we do for small $a$ ?

The function is so nice; we can just use the Taylor expansion around $a=0$. The first term is as follows.

$$
I(a) \approx a
$$

The plot for $a \in[0,1]$ is shown in Figure 2. The proof is simple. The Taylor series look like the following.

$$
I(a)=I(0)+I^{\prime}(0) a+\frac{I^{\prime \prime}(0)}{2 !} a^{2}+\frac{I^{\prime \prime \prime}(0)}{3 !} a^{3}+\cdots
$$




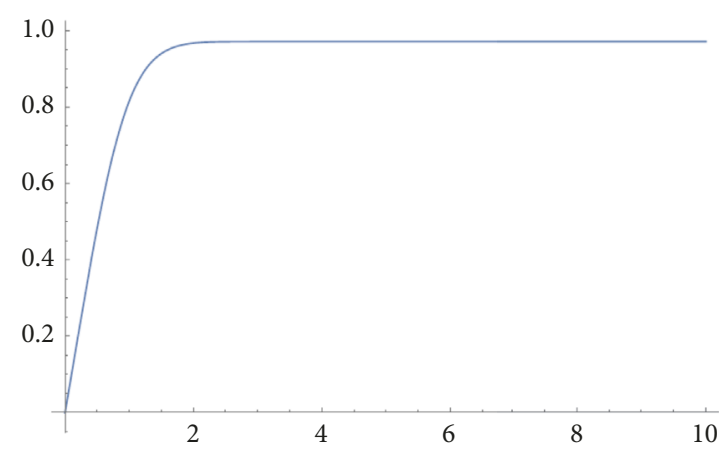

FIgURE 1: The plot of $I(a)$ for $a \in[0,10]$.

We may see the following.

$$
\begin{aligned}
I(0) & =0 \\
I^{\prime}(0) & =\left.e^{-a^{2} \operatorname{erf}(a)}\right|_{a=0}=1
\end{aligned}
$$

Now let us find a better approximation by computing the higher derivatives.

$$
\begin{aligned}
I^{\prime \prime}(a) & =\left(e^{-a^{2} \operatorname{erf}(a)}\right)^{\prime} \\
& =-\frac{2}{\sqrt{\pi}} a e^{-a^{2}(\operatorname{erf}(a)+1)}\left(\sqrt{\pi} e^{a^{2}} \operatorname{erf}(a)+a\right) \\
I^{\prime \prime}(0) & =0
\end{aligned}
$$

We use Mathematica as a shortcut, but it is easy to do it by hand, if we remember that

$$
\begin{aligned}
\operatorname{erf}^{\prime}(x) & =\frac{2}{\sqrt{\pi}} e^{-x^{2}} \\
I^{\prime \prime \prime}(0) & =0 \\
I^{I V}(0) & =-\frac{12}{\sqrt{\pi}}
\end{aligned}
$$

so our next approximation is as follows.

$$
I(a) \approx a-\frac{1}{2 \sqrt{\pi}} a^{4}
$$

The plot with both approximations (orange, green) and the function itself (blue) is given in Figure 3 and we can continue in the same way for higher derivatives. Now we admit that it is possible that we need the values of $I(a)$ for all the possible $a$ and with high precision, so the approximations will not do that. Then we need to turn to numerical integration (as Mathematica did for me to plot the function). Another way to approximate the function [2] is using its derivative:

$$
\frac{d I}{d a}=e^{-a^{2} \operatorname{erf}(a)}
$$

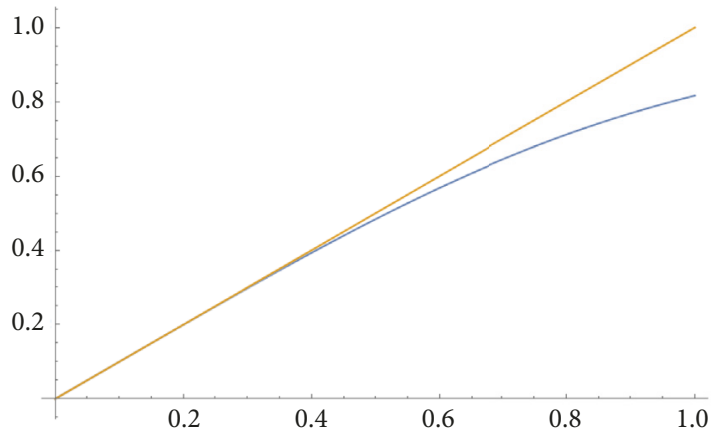

Figure 2: Approximation of $I(a)$ for $a \in[0,1]$ using Taylor expansion.

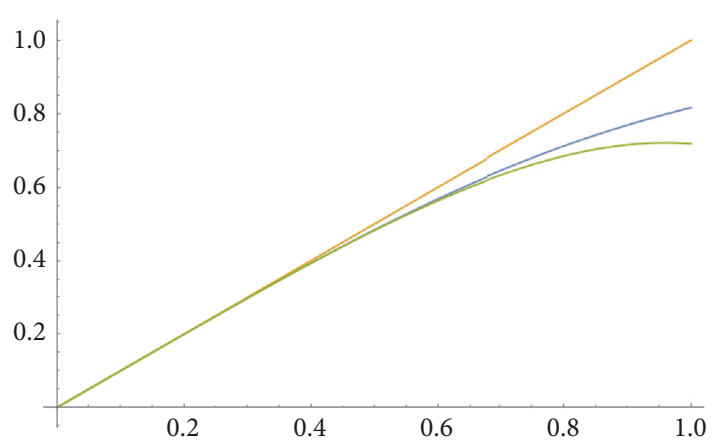

FIGURE 3: Plot of I(a) with both approximations.

but this is an ordinary differential equation, which can be solved numerically.

As an illustration, here is a simple explicit Euler scheme for the step size $h$.

$$
\begin{aligned}
\frac{I(a+h)-I(a)}{h} & =e^{-a^{2} \operatorname{erf}(a)} \\
I(a+h) & =I(a)+h e^{-a^{2} \operatorname{erf}(a)}
\end{aligned}
$$

We can use an initial value $I(0)=0$.

For $h=1 / 10$, we have the following result (red dots) compared to the exact function (blue line) as shown in Figure 4.

For $h=1 / 50$ see Figure 5 .

This way can serve as a good alternative to numerical integration [3] (depending on the context and the application of course). Let us now show the relationship between this function and other standard special functions (integral of error function) [4] as error function and cumulative distribution function for normal distribution in the context of its use. Function (1) could be used to find values of complicated integral which are not available in any references of standard special functions and also it is not available to get their values in Wolfram Alpha, for example,

$$
\int_{0}^{+\infty} e^{x^{2}(1-2 \Phi(x \sqrt{2}))} d x
$$




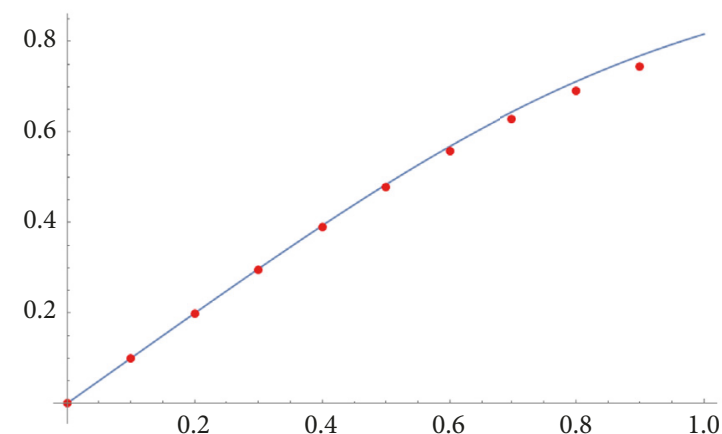

FIGURE 4: A simple explicit Euler scheme for the step size $h=1 / 10$.

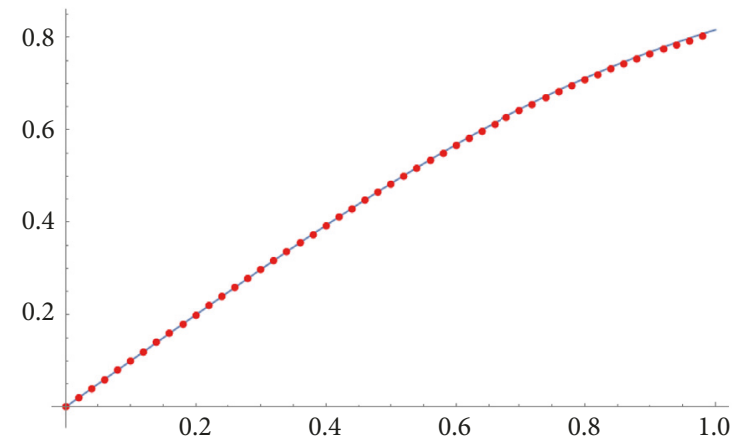

FIGURE 5: A simple explicit Euler scheme for the step size $h=1 / 50$.

with $\Phi(x)=(1 / \sqrt{2 \pi}) \int_{-\infty}^{x} e^{-z^{2} / 2} \mathrm{~d} z$. It is CDF (cumulative distribution function for normal distribution); if someone was asked to find the value of this integral, he would be confused because it is very complicated; probably he cannot show whether it is convergent or not; even Wolfram Alpha as a best means of computation cannot recognize at a least that $\phi$ is a cumulative normal distribution, so no result would be obtained about the value of this integral. Let us compute (25) using (1) and we will conclude that they have the same value and both are identical function and identical integral.

The well-known formula which expresses the relationship between error function and cumulative density function, see $\left({ }^{2}\right)$, is defined as

$$
\begin{aligned}
\operatorname{Erf}(x) & =2(\Phi(x \sqrt{2})-\Phi(0))=2\left(\Phi(x \sqrt{2})-\frac{1}{2}\right) \\
& =2 \Phi(x \sqrt{2})-1
\end{aligned}
$$

And it is easy to check that it always holds for every real number by the following short proof.

Proof. By definition, the error function

$$
\operatorname{Erf}(x)=\frac{2}{\sqrt{\pi}} \int_{0}^{x} e^{-t^{2}} \mathrm{~d} t
$$

Writing $t^{2}=z^{2} / 2$ implies $t=z / \sqrt{2}$ (because $t$ is not negative), whence $\mathrm{d} t=\mathrm{d} z / \sqrt{2}$. The endpoints $t=0$ and $t=x$ become $z=0$ and $z=x \sqrt{2}$. To convert the resulting integral into something that looks like a cumulative distribution function (CDF), it must be expressed in terms of integrals that have lower limits of $-\infty$; thus

$$
\begin{aligned}
\operatorname{Erf}(x) & =\frac{2}{\sqrt{2 \pi}} \int_{0}^{x \sqrt{2}} e^{-z^{2} / 2} \mathrm{~d} z \\
& =2\left(\frac{1}{\sqrt{2 \pi}} \int_{-\infty}^{x \sqrt{2}} e^{-z^{2} / 2} \mathrm{~d} z-\frac{1}{\sqrt{2 \pi}} \int_{-\infty}^{0} e^{-z^{2} / 2} \mathrm{~d} z\right) .
\end{aligned}
$$

Those integrals on the right hand size are both values of the CDF of the standard normal distribution:

$$
\Phi(x)=\frac{1}{\sqrt{2 \pi}} \int_{-\infty}^{x} e^{-z^{2} / 2} \mathrm{~d} z .
$$

Specifically,

$$
\begin{aligned}
\operatorname{Erf}(x) & =2(\Phi(x \sqrt{2})-\Phi(0))=2\left(\Phi(x \sqrt{2})-\frac{1}{2}\right) \\
& =2 \Phi(x \sqrt{2})-1 .
\end{aligned}
$$

Now since the LHS of (18) has a known value which is $0.97210699 \ldots$, then the right hand side also equals $0.97210699 \cdots$; hence we came up with the following identity:

$$
\int_{0}^{a}\left(e^{-x^{2}}\right)^{\operatorname{Erf}(x)} d x=\int_{0}^{a} e^{x^{2}(1-2 \Phi(x \sqrt{2}))} d x
$$

Now we shall call the function defined in (1) $T(x)=$ $\int_{0}^{X}\left(e^{-t^{2}}\right)^{\operatorname{erf}(t)} d t$ since it does not refer to anyone and it has unknown analytic representation as elementary function using standard special functions and the RHS of (18) presents another representation of $T(x)$ function using CDF of the normal distribution.

Lemma 1. $T(x)=\int_{0}^{X}\left(e^{-t^{2}}\right)^{\text {erf }(t)} d t$ cannot be expressed in terms of elementary function.

Proof. It is a theorem of Liouville [5], reproven later with purely algebraic methods, that for rational functions $f$ and $g, g$ is nonconstant, the antiderivative

$$
\int[f(x) \exp (g(x))] \mathrm{d} x
$$

can be expressed in terms of elementary functions if and only if there exists some rational function $h$ such that it is a solution to the differential equation:

$$
f=h^{\prime}+h g \text {. }
$$




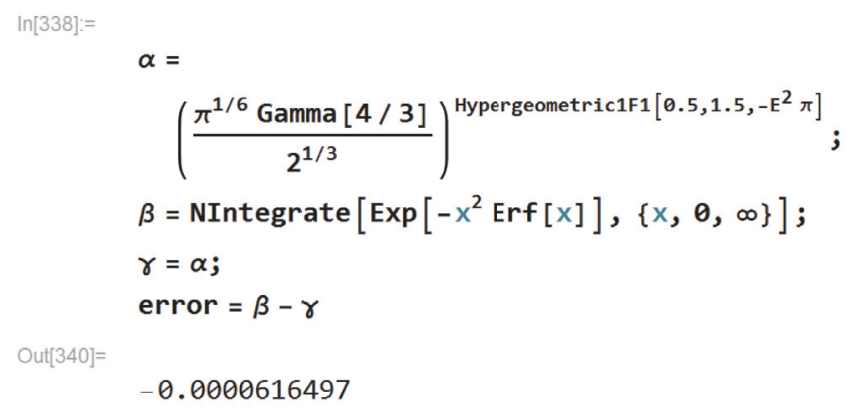

Figure 6: Error approximation for $\mathrm{T}(+\infty)$.

Now if we apply Liouville theorem we can come up with the following ODE: $1=h^{\prime}(x)+h(x)\left(-x^{2} \operatorname{erf}(x)\right)$ with $g(x)=$ $\left.-x^{2} \operatorname{erf}(x)\right)$ and $f(x)=1$. It is first ordinary differential equation. The computation we made with Wolfram Alpha gives the following solution:

$$
\begin{aligned}
& \exp \left(\frac{e^{-x^{2}}\left(e^{x^{2}}\left(\sqrt{\pi} x^{3} \operatorname{erf}(x)-1\right)+x^{2}+1\right)}{3 \sqrt{\pi}}\right) \\
& \cdot\left(e ^ { 1 / 3 \sqrt { \pi } } \left(\int_{1}^{x} \exp \left(\frac{1}{3}\left(t^{3}(-\operatorname{erf}(t))-\frac{e^{-t^{2}}\left(t^{2}+1\right)}{\sqrt{\pi}}\right)\right) d t\right.\right. \\
& \left.\left.-\int_{1}^{0} \exp \left(\frac{1}{3}\left(t^{3}(-\operatorname{erf}(t))-\frac{e^{-t^{2}}\left(t^{2}+1\right)}{\sqrt{\pi}}\right)\right) d t\right)+1\right)
\end{aligned}
$$

with $h(0)=1$. Really the function $h$ can be written follows.

$$
h(x)=l(x)\left[c_{1}+\int_{1}^{x} l(-\xi) d \xi\right]
$$

Now it is clear that $l(x)$ is a transcendental function and the defined integral in the right hand side of the $h(x)$ expression is also transcendental function because we have derivatives of rational functions being rational functions. Therefore, if the antiderivative is rational, then the original function was rational. The function $h$ is rational only at $x=0$, and since $h(x) \neq 0$, then the sum of two transcendental functions is always transcendental function. According to definition of the rational function, $h(x)$ cannot be called a rational function; then we are done.

\section{A Possible Approach Formula for $T(+\infty)$}

We may give here a possible approach formula for $\mathrm{T}(+\infty)$ which is defined as follows.

$$
\begin{aligned}
T(+\infty) & =\int_{0}^{+\infty} \exp \left(-x^{2} \operatorname{erf}(x)\right) d x \\
& =0.97210699 \cdots
\end{aligned}
$$

The inverse symbolic calculator is unable to give us the representation of 0.97210699 ... using standard special functions, but we have tried to give its representation using error function representation as hypergeometric function [6]; we have

$$
\operatorname{erf}(x)=\frac{2}{\sqrt{\pi}} x 1 F 1\left(\frac{1}{2} ; \frac{3}{2} ;-x^{2}\right)
$$

with $1 F 1$ being the Kummer confluent hypergeometric function [6]. Now we have from (24) the following.

$$
\begin{aligned}
& \int_{0}^{+\infty} \exp \left(-x^{2} \operatorname{erf}(x)\right) d x \\
& \quad=\int_{0}^{+\infty} \exp \left(-\frac{2}{\sqrt{\pi}} x^{3}\right)^{1 F 1\left(1 / 2 ; 3 / 2 ;-x^{2}\right)} d x
\end{aligned}
$$

The RHS of (25) using (24) gives $\left((\pi)^{1 / 6} \Gamma(4 / 3) / 2^{1 / 3}\right)^{1 F 1\left(0.5 ; 1.5 ;-x^{2}\right)}$. Hence we may choose $x=e \sqrt{\pi}$ and we can get finally the following.

$$
\begin{aligned}
T(+\infty) & \sim{\frac{(\pi)^{1 / 6} \Gamma(4 / 3)}{2^{1 / 3}}}^{\left.1 F 1\left(0.5 ; 1.5 ;-\pi e^{2}\right)\right)} \\
& =0.97216864 \cdots
\end{aligned}
$$

Mathematica gives the nice approximation of (25) as shown in Figure 6.

\section{Series Representation of $T(x)$ Function}

We may try to find a series expansion in powers of $t$ of

$$
I(t)=\int_{0}^{t} \exp \left(-x^{2} \operatorname{erf}(x)\right) d x=\sum_{p=1}^{\infty} c_{p} t^{p} .
$$




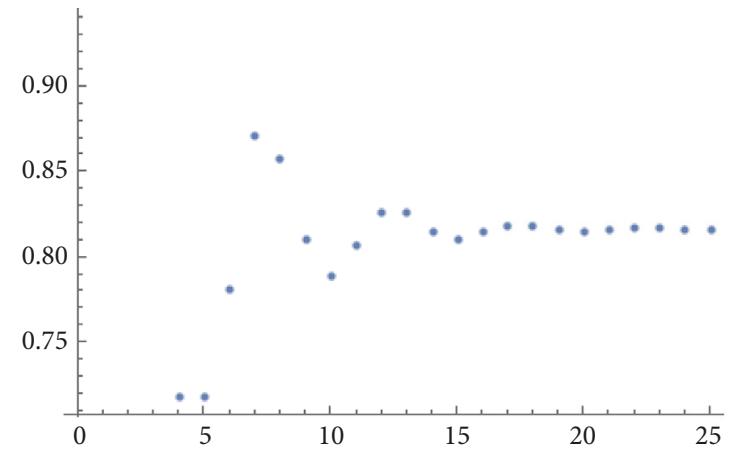

Figure 7: Convergence of $I_{N}=\sum_{p=0}^{N} c_{p}$ as a function of $N$ up to $N=25$.

The coefficients $c_{p}=p^{-1} d_{p-1}$ follow from the series expansion $e^{-x^{2} \text { erf } x}=\sum_{p=0}^{\infty} d_{p} x^{p}$, resulting in

$$
\begin{aligned}
I(t)= & \sum_{p=1}^{\infty} c_{p} t^{p} \\
= & t-\frac{t^{4}}{2 \sqrt{\pi}}+\frac{t^{6}}{9 \sqrt{\pi}}+\frac{2 t^{7}}{7 \pi}-\frac{t^{8}}{40 \sqrt{\pi}}-\frac{4 t^{9}}{27 \pi} \\
& +\frac{(\pi-28) t^{10}}{210 \pi^{3 / 2}}+O\left(t^{11}\right) .
\end{aligned}
$$

The series $I(1)=\sum_{p=1}^{\infty} c_{p}$ seems to converge. See Figure 7: The value of $I_{25}=0.8162$ agrees with $I(1)=0.816377$ to three decimal places. For $N=50$ the agreement is up to six decimal places, but this did not give us the power series closed form for $n$th term. We should use some approximations using approximation of error function and Padé approximant as shown in the following sections.

\section{Series Expansion of the $n$-th}

$$
\text { Derivative of } T(x)=\int_{0}^{x} e^{-\xi^{2} \operatorname{erf}(\xi)} d \xi
$$

Lemma 2. Series expansion of $T(x)=\int_{0}^{x} e^{-\xi^{2} \operatorname{erf}(\xi)} d \xi$ is defined by this identity:

$$
\begin{aligned}
\int_{0}^{x} e^{-\xi^{2} \operatorname{erf}(\xi) d \xi} \\
\quad=\sum_{n=0}^{\infty} \lim _{\varepsilon->0}\left(\sum_{\substack{k_{1}+2 k_{2}+\cdots+n k_{n}=n \\
k_{1} \geq 0, k_{2} \geq 0, \ldots, k_{n} \geq 0}} \prod_{j=1}^{n} \frac{A_{j, \varepsilon}^{k_{j}}}{k_{j} !}\right) \frac{x^{n+1}}{n+1}
\end{aligned}
$$

where

$$
\begin{aligned}
& A_{j, \epsilon}=\frac{2(-1)^{(j-1) / 2}}{(j-2)((1 / 2)(j-3)) ! \sqrt{\pi}} \\
& \qquad \text { if } j \geq 3 \text { and } j \text { an odd integer; } \\
& A_{j, \epsilon}=\varepsilon \quad \text { otherwise }(0<\varepsilon<1)
\end{aligned}
$$

which is the key idea to get.

Proof. Suppose that we have the Taylor expansions:

$$
f(x)=\sum_{n=1}^{\infty} \frac{a_{n}}{n !} x^{n}
$$

and

$$
g(x)=\sum_{n=1}^{\infty} \frac{b_{n}}{n !} x^{n}
$$

Then we have the standard result:

$$
g(f(x))=\sum_{n=1}^{\infty}\left(\sum_{k=1}^{n} b_{k} B_{n, k}\left(a_{1}, \ldots, a_{n-k+1}\right)\right) \frac{x^{n}}{n !}
$$

where $B_{n, k}(\cdot)$ are the partial Bell polynomials, which are defined by the following formula.

$$
\begin{aligned}
& \widehat{B}_{m, j}\left(x_{1}, x_{2}, \ldots, x_{m-j+1}\right) \\
& =\sum_{\substack{k_{0}+k_{1}+\cdots+k_{N}=j \\
k_{1}+2 k_{2}+\cdots+N k_{N}=m}}\left(\begin{array}{c}
j \\
k_{0}, k_{1}, \ldots, k_{N}
\end{array}\right) \prod_{i=1}^{N} x_{i}^{k_{i}}
\end{aligned}
$$

The key idea to get series expansion of the $n$-th derivative of $T(x)$ which is defined in (29) is to use Taylor expansion of $g(x)=\exp (-x)$ and $f(x)=-x^{2} \operatorname{erf}(x)$ coming up for using one of the important formulas in mathematics called BrunoFadi formula such as that defined above in (33) using (34). It is well known that the Taylor expansion of $\exp (-x)$ is given by the following.

$$
g(x)=\exp (-x)=\sum_{n=0}^{\infty} \frac{(-1)^{n} x^{n}}{n !}
$$

Probably the interesting here for readers to know is Taylor expansion of $\operatorname{erf}(x)$; we give a simple proof about its expansion series using Hermite polynomial.

Lemma 3. Taylor expansion of $\operatorname{erf}(x)$ at each point a is given by this identity:

$$
\operatorname{erf}_{a}(x)=e^{-a^{2}} \sum_{n=0}^{\infty}(-1)^{n} \frac{H_{n}(a)}{n !}(x-a)^{n}
$$

with $H_{n}(a)$ is Hermite polynomial of degree $n$. 
Proof. $f^{(n)}(a)$ can be written in terms of Hermite polynomials $H_{n}$.

$$
\begin{aligned}
& H_{0}(x)=1, \\
& H_{1}(x)=2 x, \\
& H_{2}(x)=4 x^{2}-2, \\
& H_{3}(x)=8 x^{3}-12 x, \\
& H_{4}(x)=16 x^{4}-48 x^{2}+12, \\
& H_{5}(x)=\cdots
\end{aligned}
$$

We may recognize that $H_{2 n-1}(0)=0$, which gives the power series for $e^{-x^{2}}$ at $a=0$.

$$
e^{-x^{2}}=1-\frac{2}{2 !} x^{2}+\frac{12}{4 !} x^{4}-\frac{120}{6 !} x^{6}+\cdots
$$

After multiplying by $2 / \sqrt{\pi}$, this integrates to

$$
\operatorname{erf}(z)=\frac{2}{\sqrt{\pi}}\left(z-\frac{z^{3}}{3}+\frac{z^{5}}{10}-\frac{z^{7}}{42}+\frac{z^{9}}{216}-\cdots\right)
$$

Since $\left(d^{n} / d x^{n}\right) e^{-x^{2}}=(-1)^{n} e^{-x^{2}} H_{n}(x)$, one can do a Taylor Series for every $a$.

$$
\operatorname{erf}_{a}(x)=e^{-a^{2}} \sum_{n=0}^{\infty}(-1)^{n} \frac{H_{n}(a)}{n !}(x-a)^{n}
$$

Then we are done.

Now by composition of (40) with (35) after multiplying (40) by the term $-x^{2}$, we come up to Bruno-Fadi formula which is defined as

$$
e^{-x^{2} \operatorname{erf}(x)} \sum_{n=0}^{\infty} \lim _{\varepsilon \rightarrow 0}\left(\sum_{\substack{k_{1}+2 k_{2}+\cdots+n k_{n}=n \\ k_{1} \geq 0, k_{2} \geq 0, \ldots, k_{n} \geq 0}} \prod_{j=1}^{n} \frac{A_{j, \varepsilon}^{k_{j}}}{k_{j} !}\right) x^{n},
$$

where

$$
\begin{aligned}
& A_{j, \epsilon}=\frac{2(-1)^{(j-1) / 2}}{(j-2)((1 / 2)(j-3)) ! \sqrt{\pi}} \\
& \quad \text { if } j \geq 3 \text { and } j \text { an odd integer; } \\
& A_{j, \epsilon}=\varepsilon \quad \text { otherwise }(0<\varepsilon<1) .
\end{aligned}
$$

Integrating this equation term by term gives

$$
\begin{aligned}
\int_{0}^{x} e^{-\xi^{2} \operatorname{erf}(\xi) d \xi} \\
\quad=\sum_{n=0}^{\infty} \lim _{\varepsilon->0}\left(\sum_{\substack{k_{1}+2 k_{2}+\cdots+n k_{n}=n \\
k_{1} \geq 0, k_{2} \geq 0, \ldots, k_{n} \geq 0}} \prod_{j=1}^{n} \frac{A_{j, \varepsilon}^{k_{j}}}{k_{j} !}\right) \frac{x^{n+1}}{n+1}
\end{aligned}
$$

which gives the series expansion for the new special function. Using Mathematica as a shortcut, it shows that (44) holds and also it shows the incrementation of $\pi$ as shown in Figure 8 . We may add also the series expansion of $T(x)$, the new special function, using Mathematica code as shown in Figure 9.

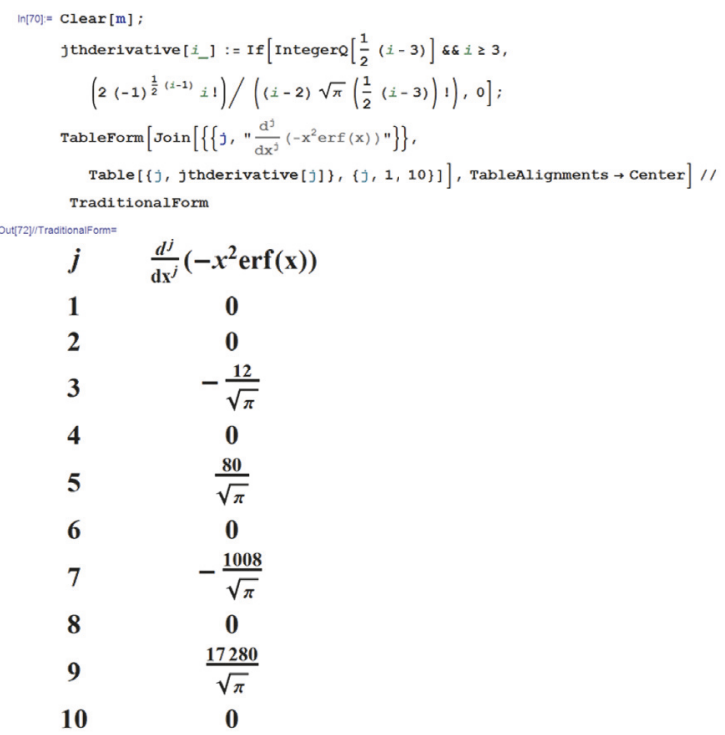

FIGURE 8: n-th derivative of new special function $T(x)$.

$$
\begin{aligned}
& \operatorname{In}[140]=\quad \begin{array}{l}
\text { Clear }[x] ; \\
\text { Sum }\left[\operatorname{coeff}[j] * x^{j},\{j, 0,12\}\right], \\
\text { Normal } \left.\left[\operatorname{Series}\left[\operatorname{Exp}\left[-x^{2} \operatorname{Erf}[x]\right],\{x, 0,12\}\right]\right]\right\} / / \text { Simplify }
\end{array} \\
& \left\{\frac { 1 } { 3 7 8 0 \pi ^ { 2 } } \left(3780 \pi^{2}+2520 x^{12}+5040 \sqrt{\pi} x^{9}\left(-1+x^{2}\right)-\right.\right. \\
& 24 \pi x^{6}\left(-315+210 x^{2}-98 x^{4}+36 x^{6}\right)+ \\
& \left.\pi^{3 / 2} x^{5}\left(-7560+2520 x^{2}-756 x^{4}+180 x^{6}-35 x^{8}\right)\right), \\
& \frac{1}{3780 \pi^{2}}\left(3780 \pi^{2}+2520 x^{12}+5040 \sqrt{\pi} x^{9}\left(-1+x^{2}\right)-\right. \\
& 24 \pi x^{6}\left(-315+210 x^{2}-98 x^{4}+36 x^{6}\right)+ \\
& \left.\left.\pi^{3 / 2} x^{3}\left(-7560+2520 x^{2}-756 x^{4}+180 x^{6}-35 x^{8}\right)\right)\right\}
\end{aligned}
$$

FIGURE 9: Series expansion of new special function $T(x)$ around $x=$ 0 .

\section{Series Representation of $\int_{-1}^{1} e r f(x)^{n} d x$ Using Error Function Approximation}

We have the power series of

$$
e^{-x^{2} \operatorname{erf}(x)}=\sum_{k=0}^{\infty} \frac{(-1)^{k} x^{2 k} \operatorname{erf}^{k}(x)}{k !}
$$

and then from (45) we have the following.

$$
\int_{-1}^{1} e^{-x^{2} \operatorname{erf}(x)} d x=\sum_{k=0}^{\infty} \frac{(-1)^{k}}{k !} \int_{-1}^{1} x^{2 k} \operatorname{erf}^{k}(x) d x
$$

Now it is hard so much to evaluate the integral in RHS of (46) using error function expression; then we should use the following nice approximation.

$$
(\operatorname{erf}(x))^{2} \approx 1-e^{-a x^{2}} \quad \text { with } a=(1+\pi)^{2 / 3} \log ^{2}(2)
$$

Here we can give a short proof to show that the error function squared was approximated as well with the value of $a=(1+$ $\pi)^{2 / 3} \log ^{2}(2)$ 
Proof. We fully agree that

$$
F(a)=\int_{0}^{\infty}\left(\operatorname{erf}(x)^{2}-\left(1-e^{-a x^{2}}\right) d x\right)^{2}
$$

is minimum for $a \approx 1.23907$. According to RIES, this number seems to be much closer to

$$
a=(1+\pi)^{2 / 3} \log ^{2}(2) \approx 1.23907
$$

than to $\pi^{2} / 8 \approx 1.23370$ even if this does make very large difference (the maximum error is reduced from 0.006 to 0.004 and the value of the integral $F(a)$ changes from 0.00002769 to 0.00002572 ). If we look for a still better approximation, we could consider $\log \left(1-\operatorname{erf}(x)^{2}\right)$ (which, for sure, introduces a bias in the problem), establish a Padé approximant, and finally arrive to

$$
\operatorname{erf}(x)^{2} \approx 1-\exp \left(-\frac{4}{\pi} \frac{1+\alpha x^{2}}{1+\beta x^{2}} x^{2}\right)
$$

where

$$
\begin{aligned}
& \alpha=\frac{10-\pi^{2}}{5(\pi-3) \pi} \\
& \beta=\frac{120-60 \pi+7 \pi^{2}}{15(\pi-3) \pi} .
\end{aligned}
$$

The value of the corresponding error function is $1.1568 \times 10^{-7}$, that is to say, almost 250 times smaller than that with the initial formulation; the maximum error is 0.00035 .

Now we are ready to approximate

$$
\begin{aligned}
& I_{n}=\int_{-1}^{1}(\operatorname{erf}(x))^{2 n} d x \\
& J_{n}=\int_{-1}^{1}\left(1-e^{-a x^{2}}\right)^{n} d x
\end{aligned}
$$

for which the binomial expansion would be required (easy). This would give you things like the following.

$$
\begin{gathered}
J_{1}=2-\frac{\sqrt{\pi} \operatorname{erf}(\sqrt{a})}{\sqrt{a}} \\
J_{2}=2-\frac{2 \sqrt{\pi} \operatorname{erf}(\sqrt{a})}{\sqrt{a}}+\frac{\sqrt{\pi / 2} \operatorname{erf}(\sqrt{2 a})}{\sqrt{a}} \\
J_{3}=2-\frac{3 \sqrt{\pi} \operatorname{erf}(\sqrt{a})}{\sqrt{a}}+\frac{3 \sqrt{\pi / 2} \operatorname{erf}(\sqrt{2 a})}{\sqrt{a}} \\
-\frac{\sqrt{\pi / 3} \operatorname{erf}(\sqrt{3 a})}{\sqrt{a}}
\end{gathered}
$$

Now it is easy to get recurrence relation for $J_{n}$ in (53); we take $t=\sqrt{a k} x \Longrightarrow d x=d t /(\sqrt{a k})$ and we come up to $\operatorname{erf}(\sqrt{a k})$ which gives the following general formula.

$$
J_{n}=2+\sqrt{\frac{\pi}{a}} \sum_{k=1}^{n}(-1)^{k} \frac{\left(\begin{array}{l}
n \\
k
\end{array}\right)}{\sqrt{k}} \operatorname{erf}(\sqrt{a k})
$$

TABLE 1: Short table for approximation comparison.

\begin{tabular}{lcc}
\hline $\mathrm{n}$ & approximation & exact \\
\hline 1 & 0.591506 & 0.596751 \\
2 & 0.279674 & 0.283168 \\
3 & 0.151067 & 0.153256 \\
4 & 0.0870954 & 0.0884650 \\
5 & 0.0522216 & 0.0530855 \\
6 & 0.0321485 & 0.0326982 \\
7 & 0.0201718 & 0.0205243 \\
8 & 0.0128409 & 0.0130686 \\
9 & 0.00826756 & 0.00841548 \\
10 & 0.00537202 & 0.00546863 \\
\hline
\end{tabular}

We produce in Table 1 a short table for comparison; we reused for this problem our approach with the same Padé approximants and obtained the following as approximations.

$$
\begin{aligned}
I_{n} & =\frac{2}{2 n+1}\left(\frac{4}{\pi}\right)^{n}{ }_{2} F_{1}\left(2 n, \frac{2 n+1}{2} ; \frac{2 n+3}{2} ;-\frac{1}{3}\right) \\
I_{n} & =\frac{2}{2 n+1}\left(\frac{4}{\pi}\right)^{n} \\
& \cdot F_{1}\left(\frac{2 n+1}{2} ;-2 n, 2 n ; \frac{2 n+3}{2} ; \frac{1}{30},-\frac{3}{10}\right)
\end{aligned}
$$

Really we are ready to give the series representation of $T(x)$ over $[-1 ; 1]$ using error function approximation and Padé approximant.

\section{Series Representation of $T(x)$ Function over $[-1 ; 1]$ Using Error Function Approximation and Padé Approximant}

Recall

$$
I_{k}=\int_{-1}^{1} x^{2 k}[\operatorname{erf}(x)]^{k} d x
$$

is 0 if $k$ is odd. Thus, we need to focus on

$$
I_{2 k}=\int_{-1}^{1} x^{4 k}[\operatorname{erf}(x)]^{2 k} d x
$$

which could be approximated, as we showed above in Section 3 to get (57) using

$$
[\operatorname{erf}(x)]^{2} \approx 1-e^{-a x^{2}} \quad \text { with } a=(1+\pi)^{2 / 3} \log ^{2}(2)
$$

making

$$
I_{2 k}=\int_{-1}^{1} x^{4 k}\left(1-e^{-a x^{2}}\right)^{k} d x
$$

to be developed using the binomial expansion. Therefore, in practice, we face the problem of

$$
J_{n, k}=\int_{-1}^{1} x^{4 k} e^{-n a x^{2}} d x
$$


TABLE 2: Reasonable approximation using bounds.

\begin{tabular}{lcc}
\hline $\mathrm{k}$ & approximation & exact \\
\hline 1 & 0.22870436048 & 0.22959937502 \\
2 & 0.08960938943 & 0.08997882179 \\
3 & 0.04400808083 & 0.04418398568 \\
4 & 0.02389675159 & 0.02398719298 \\
5 & 0.01374034121 & 0.01378897319 \\
6 & 0.00819869354 & 0.00822557475 \\
7 & 0.00502074798 & 0.00503586007 \\
8 & 0.00313428854 & 0.00314286515 \\
9 & 0.00198581489 & 0.00199069974 \\
10 & 0.00127304507 & 0.00127582211 \\
\hline
\end{tabular}

and the antiderivative

$$
\int x^{4 k} e^{-n a x^{2}} d x=-\frac{1}{2} x^{4 k+1} E_{1 / 2-2 k}\left(a n x^{2}\right)
$$

where the exponential integral function appears. Using the bounds, this reduces to

$$
J_{n, k}=-E_{1 / 2-2 k}(a n)
$$

and leads to "reasonable" approximation as shown in Table 2. Another approximation could be obtained using the simplest Padé approximant [7] of the error function

$$
\operatorname{erf}(x)=\frac{2 x}{\sqrt{\pi}\left(1+x^{2} / 3\right)}
$$

which would lead to

$$
\begin{aligned}
I_{2 k} & =\int_{-1}^{1} x^{4 k}[\operatorname{erf}(x)]^{2 k} d x \\
& =\frac{2}{6 k+1}\left(\frac{4}{\pi}\right)^{k}{ }_{2} F_{1}\left(2 k, \frac{6 k+1}{2} ; \frac{6 k+3}{2} ;-\frac{1}{3}\right)
\end{aligned}
$$

slightly less accurate than the previous one. Continuing with Padé approximant

$$
\operatorname{erf}(x)=\frac{2 x / \sqrt{\pi}-x^{3} / 15 \sqrt{\pi}}{1+3 x^{2} / 10}
$$

we should get

$$
\begin{aligned}
I_{2 k} & =\int_{-1}^{1} x^{4 k}[\operatorname{erf}(x)]^{2 k} d x=\frac{2}{6 k+1}\left(\frac{4}{\pi}\right)^{k} \\
& \cdot F_{1}\left(\frac{6 k+1}{2} ;-2 k, 2 k ; \frac{6 k+3}{2} ; \frac{1}{30},-\frac{3}{10}\right)
\end{aligned}
$$

where the Appell hypergeometric function of two variables appears. Finally we conclude the series representation as follows.

$$
I(t)=\int_{-1}^{1} \exp \left(-x^{2} \operatorname{erf}(x)\right) d x \cdot \sim \sum_{k=0}^{+\infty} \frac{(-1)^{k}}{k !} I_{2 k}
$$

\section{Approximation of $T(x)$ Function by Means of a Polynomial}

Lemma 4. The function $f$ which is defined as

$$
f(x)=T\left(\frac{b+a}{2}+\frac{b-a}{2} x\right), \quad-1 \leq x \leq 1
$$

could be approximated by means of Chebytchev polynomial.

Proof. We may approximate the function $f$ on the interval $[-1,1]$ by using Chèbyshev polynomials [8] of the first kind. To this end, we choose some positive integer $n$ and we define the coefficients $c_{n}$ by the formula

$$
c_{j}=\frac{2}{\pi} \int_{-1}^{1} \frac{T_{j}(x)}{\sqrt{1-x^{2}}} f(x) d x \text { for } j=0,1, \ldots, n .
$$

Then the polynomial

$$
P_{n}(x)=\frac{1}{2} c_{0}+\sum_{j=1}^{n} c_{j} T_{j}(x)
$$

approximates $f(x)$ in the best possible way. Since

$$
T(x)=f\left(\frac{a+b-2 x}{a-b}\right) \quad \text { for } a \leq x \leq b
$$

we see that the polynomial $Q_{n}(a, b, x)=P_{n}((a+b-2 x) /(a-$ $b))$ is an approximant to $T(x)$ function on $[a, b]$. Calculations give

$$
\begin{aligned}
Q_{11}\left(0, \frac{3}{2}, x\right)= & 0.0137936039435 x^{11} \\
& -0.135129528505 x^{10} \\
& +0.548169602543 x^{9} \\
& -1.16161653976 x^{8} \\
& +1.31691631085 x^{7} \\
& -0.746480407376 x^{6} \\
& +0.338453415662 x^{5} \\
& -0.370071852413 x^{4} \\
& +0.0133517048763 x^{3} \\
& -0.00104123958376 x^{2} \\
& +1.00003172454 x
\end{aligned}
$$


and

$$
\begin{aligned}
Q_{11}\left(\frac{3}{2}, 3, x\right)= & -0.0000675632422240 x^{11} \\
& +0.00188305739843 x^{10} \\
& -0.0239397852528 x^{9} \\
& +0.183255163671 x^{8} \\
& -0.937675010268 x^{7} \\
& +3.35913844398 x^{6} \\
& -8.55140470408 x^{5} \\
& +15.3046428836 x^{4} \\
& -18.4622672665 x^{3} \\
& +13.5920479951 x^{2} \\
& -4.69093970289 x \\
& +1.04191571066 .
\end{aligned}
$$

For both approximations the error is less than $10^{-6}$. Indeed, numerical integration gives

$$
\begin{aligned}
\| T & (x)-Q_{11}\left(0, \frac{3}{2}, x\right) \| \\
& =\sqrt{\int_{0}^{3 / 2}\left(T(x)-Q_{11}\left(0, \frac{3}{2}, x\right)\right)^{2} d x} \\
& \approx 2.26 \times 10^{-7}
\end{aligned}
$$

and

$$
\begin{aligned}
\| T & (x)-Q_{11}\left(\frac{3}{2}, 3, x\right) \| \\
& =\sqrt{\int_{3 / 2}^{3}\left(T(x)-Q_{11}\left(\frac{3}{2}, 3, x\right)\right)^{2} d x} \\
& \approx 3.66 \times 10^{-10} .
\end{aligned}
$$

Thus, we may evaluate the $T(x)$ function with high accuracy on the interval $[0,3]$. For $x>3$ we may use the following approximation formula in terms of the error function:

$$
\begin{array}{r}
T(x) \approx \varphi(x) \\
\stackrel{d e f}{=} \int_{0}^{3} \exp \left(-t^{2} \operatorname{erf}(t)\right) d t+\frac{\sqrt{\pi}}{2}(\operatorname{erf}(x)-\operatorname{erf}(3)), \\
x \geq 3 .
\end{array}
$$

The quadratic mean error on $[3,100]$ is

$$
\begin{aligned}
\| T(x)-\varphi(x)) \| & =\sqrt{\int_{3}^{100}(T(x)-\varphi(x))^{2} d x} \\
& \approx 2.02 \times 10^{-8} .
\end{aligned}
$$

Now we are ready to present application of $T(x)$ in probability and thermodynamics using one of the most important distributions which is called Maxwell-Boltzmann distribution

\section{Application of $T(x)$ in Probability}

Let

$$
F_{\lambda, \mu}(x)=\int_{0}^{x} e^{-\xi^{2}(\lambda+\mu \operatorname{erf}(\xi))} d \xi \quad(\lambda>0) .
$$

Define

$$
c=\int_{0}^{\infty} e^{-\xi^{2}(\lambda+\mu \operatorname{erf}(\xi))} d \xi
$$

and let

$$
T_{\lambda, \mu}(x):=c^{-1} F_{\lambda, \mu}(x) \quad(x \geq 0) .
$$

The function $T(x)$ is the new special function we have studied in this paper. This function defines a cumulative probability distribution function (CDF) with probability distribution function (PDF).

$$
f_{\lambda, \mu}(x)=e^{-x^{2}(\lambda+\mu \operatorname{erf}(x))} \quad(x \geq 0)
$$

Indeed, we have

$$
\begin{aligned}
T_{\lambda, \mu}^{\prime}(x) & =e^{-x^{2}(\lambda+\mu \operatorname{erf}(x))}>0, \\
T_{\lambda, \mu}(+\infty) & =1 .
\end{aligned}
$$

The ODE for this function not involving the error function erf may be obtained by differentiating (84) twice and eliminating the expression containing that error function. This gives us the following ODE.

$$
\sqrt{\pi} x y^{\prime \prime}(x)=2 e^{-x^{2}} y^{\prime}(x)\left(\sqrt{\pi} e^{x^{2}} \log \left(y^{\prime}(x)\right)-\mu x^{3}\right)
$$

Letting $\mu=0$ gives the $\mathrm{ODE}$

$$
x y^{\prime \prime}(x)=2 y^{\prime}(x) \log \left(y^{\prime}(x)\right)
$$

whose general solution is as follows.

$$
y(x)=\frac{1}{2} e^{-c_{1} / 2} \sqrt{\pi} \operatorname{erfi}\left(e^{c_{1} / 2} x\right)+c_{2}
$$

If we compare (89) with

$$
\begin{aligned}
c F_{\lambda, 0}(x) & =c \int_{0}^{x} e^{-\lambda \xi^{2}} d \xi \\
& =\left(\int_{0}^{\infty} e^{-\lambda \xi^{2}} d \xi\right)^{-1} \int_{0}^{x} e^{-\lambda \xi^{2}} d \xi=\operatorname{erf}(\sqrt{\lambda} x)
\end{aligned}
$$

we must have

$$
\frac{1}{2} e^{-c_{1} / 2} \sqrt{\pi} \operatorname{erfi}\left(e^{c_{1} / 2} x\right)+c_{2}=\operatorname{erf}(\sqrt{\lambda} x)
$$


so that

$$
\begin{aligned}
& c_{1}=i \pi+\log \left(\frac{\pi}{4}\right), \\
& c_{2}=0 .
\end{aligned}
$$

We showed that in the case when $\mu=0$ our function $T_{\lambda, 0}(x)$ coincides with the error function $\operatorname{erf}(\sqrt{\lambda} x)$ with the value $\lambda=\pi / 4$. When $\mu \neq 0$ we cannot obtain the solution to the ODE (87) in closed form. We may try a numerical procedure or another method to solve it. Our aim is to show how we may apply the new special function $T_{\lambda, \mu}(x)$ in probability and physics.

8.1. Example. We look for $\lambda$ and $\mu$ in order to adjust the error function by means of the function $y(x)=T_{\lambda, \mu}(x)$. To this end, we impose the following conditions.

$$
\begin{array}{r}
\operatorname{erf}(1)=T_{\lambda, \mu}(x), \\
\operatorname{erf}^{\prime}(1)=T_{\lambda, \mu}^{\prime}(1)
\end{array}
$$

Solving this system gives

$$
\begin{aligned}
\lambda & =0.1671645, \\
\mu & =0.8449657 .
\end{aligned}
$$

The function $T_{\lambda, \mu}(x)$ converts into

$$
\begin{aligned}
& T_{\lambda, \mu}(x) \\
& =1.05021 \int_{0}^{x} \exp \left(-\xi^{2}(0.167164+0.844966 \operatorname{erf}(\xi))\right) d \xi .
\end{aligned}
$$

Plotting the two functions gives following picture as shown in Figure 10

\section{Application of $T(x)$ in Thermodynamics}

In physics (in particular in statistical mechanics), the Maxwell-Boltzmann distribution is a particular probability distribution named after James Clerk Maxwell and Ludwig Boltzmann. It was first defined and used for describing particle speeds in idealized gases, where the particles move freely inside a stationary container without interacting with one another, except for very brief collisions in which they exchange energy and momentum with each other or with their thermal environment. The term "particle" in this context refers to gaseous particles (atoms or molecules), and the system of particles is assumed to have reached thermodynamic equilibrium. The energies of such particles follow what is known as Maxwell-Boltzmann statistics, and the statistical distribution of speeds is derived by equating particle energies with kinetic energy. Mathematically, the Maxwell-Boltzmann distribution is the chi distribution with three degrees of freedom (the components of the velocity vector in Euclidean space), with a scale parameter measuring speeds in units proportional to the square root of $T / m$ (the ratio of temperature and particle mass); see Figure 10. The

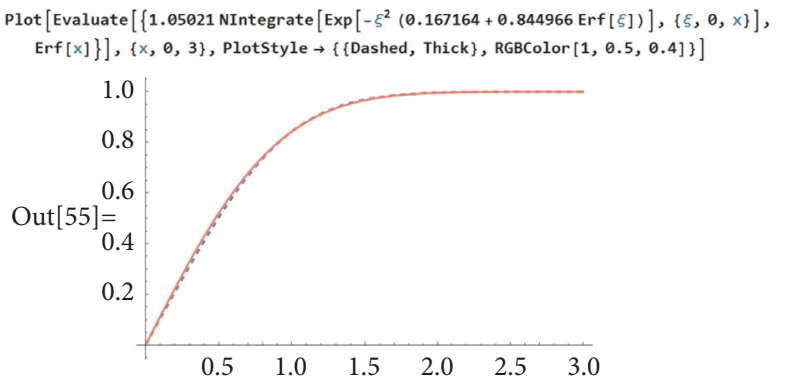

FIGURE 10: Adjusting the error function by means of the new special function.

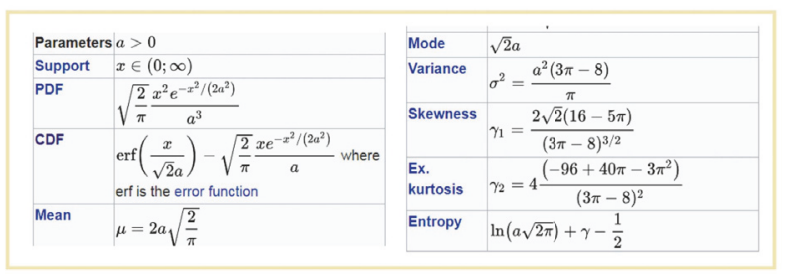

Figure 11: Maxwell-Boltzmann distribution.

$\mathrm{CDF}$ for the Boltzmann distribution may be approximated by means of the new special function $T_{\kappa, \mu}(x)$ as follows:

$$
\begin{aligned}
& \operatorname{erf}\left(\frac{x}{\sqrt{2} a}\right)-\sqrt{\frac{2}{\pi}} \frac{x}{a} \exp \left(-\frac{x^{2}}{2 a^{2}}\right) \\
& \approx T_{\lambda, \mu}(x)-\sqrt{\frac{2}{\pi}} \frac{x}{a} \exp \left(-\frac{x^{2}}{2 a^{2}}\right)
\end{aligned}
$$

where $T_{\lambda, \mu}(x)$ is an approximation to $\operatorname{erf}(x / \sqrt{2} a)$ for some parameters $\lambda$ and $\mu$ depending on $a$. This approximation may be obtained in a similar way to what we illustrated in Example 1 , Figure 11. On the other hand, in the case when $0<a \leq 1$ we may approximate the CDF for the Maxwell-Boltzmann distribution as shown in Figure 12 for the value $a=0.75$.

Finally this approximation by new special function showed that it may also be applied in thermodynamics to evaluate the average energy per particle in the circumstance where there is no energy-dependent density of states to skew the distribution, and the representation of probability for a given energy must be normalized to a probability of 1 which holds using our new special function with two parameters as shown in (86).

\section{Conclusion}

We have studied A new probability distribution defined on $[0,+\infty)$ and we gave series representations for $T(x)$ function using Padé approximant. Really we approximated the CDF for that distribution by means of Chèbyshev polynomials and the error function. The methods we applied are suitable for approximating other $\mathrm{CDF}$ for probability distributions, since their $\mathrm{CDF}$ are bounded and they take values from 0 to 1 . And it is well known that Chèbyshev polynomials are the optimal 


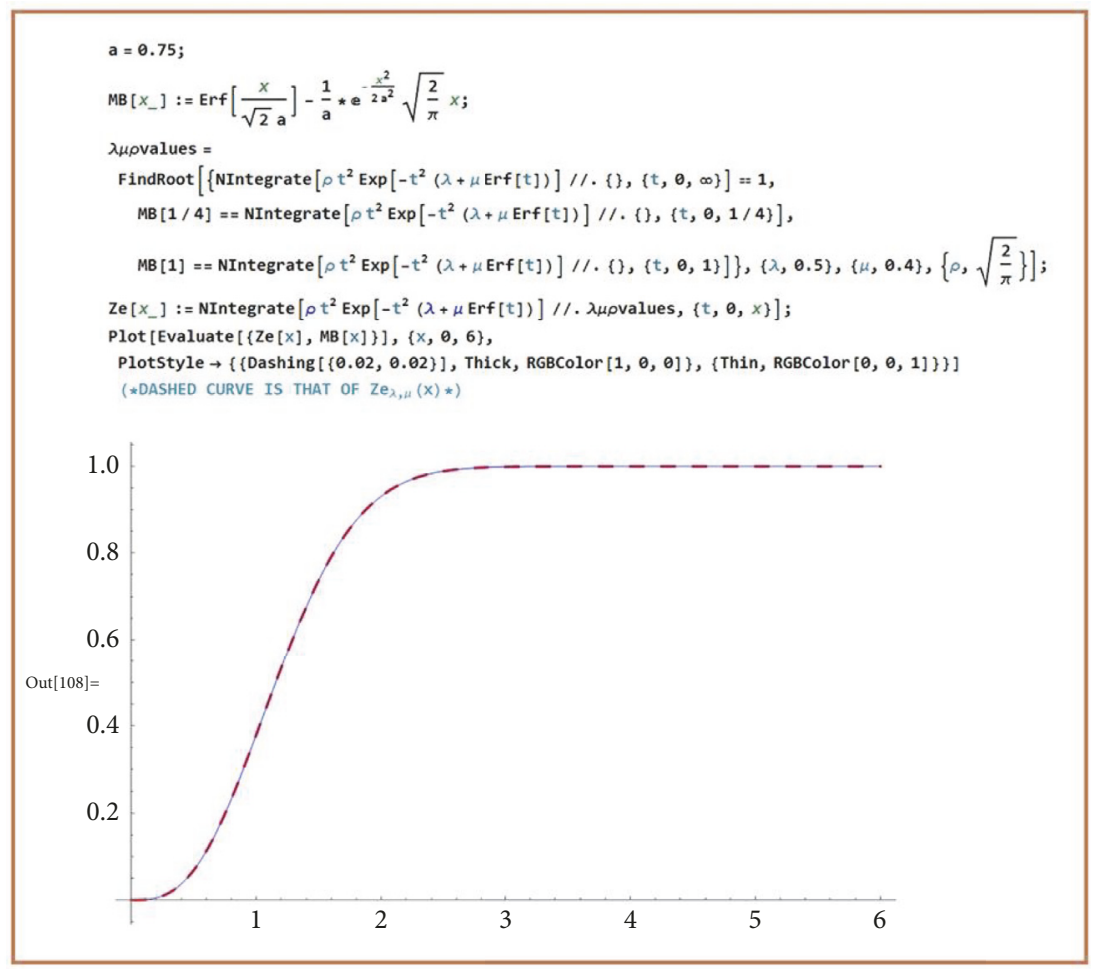

FIGURE 12: Approximation of the CDF for the Maxwell-Boltzmann distribution for $a=0.75$.

ones for approximating continuous functions. On the other hand, it is also possible to approximate such functions by means of rational Chèbyshev approximants. This technique may be used in future works.

\section{Data Availability}

The data supporting this new special function is the Table of integral of error functions which is cited as one of the important reference in this paper and that function dosn't montioned in any standard references related to the integral of error function.

\section{Disclosure}

Zeraoulia Rafik present address is Department of Mathematics, High school-Timgad, Batna, Algeria.

\section{Conflicts of Interest}

There are no conflicts of interest regarding the publication of this paper.

\section{Authors' Contributions}

Zeraoulia Rafik, Alvaro H. Salas, and David L. Ocampo are equally contributing authors.

\section{Acknowledgments}

Alvaro H. Salas, Universidad Nacional de Colombia, Colombia, has received the Grant/Award Number 283, http:// agenciadenoticias.unal.edu.co/detalle/article/profesor-de-laun-gano-premio-scopus-en-el-area-de-matematicas.html. We would like to express our deep gratitude to Professor David L. Ocampo for improving the quality of the paper. My great salutation to Professor Alvaro H. Salas and to my parents and all my great salutations to my wife and to my second heart my son Taha Abd-Aldjalil. We would also like to thank Yuriy $\mathbf{S}$ and Claude Leibovici from Stack Exchange Math and Prof. Carlo Beenakker from MathOverflow for their contributions to the paper.

\section{Endnotes}

1. In mathematics, the error function (also called the Gauss error function) is a special function (nonelementary) of sigmoid shape that occurs in probability, statistics, and partial differential equations describing diffusion. It is defined as $\operatorname{Erf}(x)=(2 / \sqrt{\pi}) \int_{0}^{x} e^{-t^{2}} d t$. Of course, it is closely related to the normal CDF $\Phi(x)=P(N<x)=$ $(1 / \sqrt{2 \pi}) \int_{-\infty}^{x} e^{-t^{2} / 2} d t$ (where $N \sim N(0,1)$ is a standard normal) by the expression $\operatorname{Erf}=2 \Phi(x \sqrt{2})-1$.

2. Cumulative distribution function for the normal distribution. In probability theory and statistics, the cumulative distribution function (CDF, also cumulative density 
function) of a real-valued random variable $X$, or just distribution function of $X$, evaluated at $x$, is the probability that $X$ will take a value less than or equal to $x$.If we have a quantity $A$ that takes some value at random, the cumulative density function $F(x)$ gives the probability that $X$ is less than or equal to $x$; that is,

$$
F(x)=P(A \leq x)
$$

In the case of a continuous distribution, it gives the area under the probability density function from minus infinity to $x$. Cumulative distribution functions are also used to specify the distribution of multivariate random variables.

\section{References}

[1] J. L. Teugels and B. Sundt, Encyclopedia of Actuarial Science, vol. 1, Wiley, 2004.

[2] N. Mai-Duy and T. Tran-Cong, "Approximation of function and its derivatives using radial basis function networks," Applied Mathematical Modelling, vol. 27, no. 3, pp. 197-220, 2003.

[3] T. Suzuki and T. Suzuki, "Numerical integration error method for zeros of analytic functions," Journal of Computational and Applied Mathematics, vol. 152, no. 1-2, pp. 493-505, 2003.

[4] E. W. Ng and M. Geller, "A table of integrals of the error functions," Journal of Research of the National Bureau of Standards, vol. 73B, pp. 1-20, 1969.

[5] J. Liouville, "Suite du Mémoire sur la classification des Transcendantes, et sur l'impossibilité d'exprimer les racines de certaines équations en fonction finie explicite des coefficients," Journal de Mathématiques Pures et Appliquées, vol. 3, pp. 523$546,1838$.

[6] https://arxiv.org/pdf/1702.08438.pdf.

[7] A. Khani and S. Shahmorad, "An operational approach with Pade approximant for the numerical solution of non-linear Fredholm integro-differential equations," Scientia Iranica, vol. 19, no. 6, pp. 1691-1698, 2012.

[8] H. N. Soloklo and M. M. Farsangi, "Chebyshev rational functions approximation for model order reduction using harmony search," Scientia Iranica, vol. 20, no. 3, pp. 771-777, 2013. 


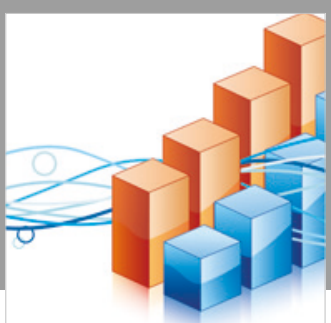

Advances in

Operations Research

\section{-n-m}
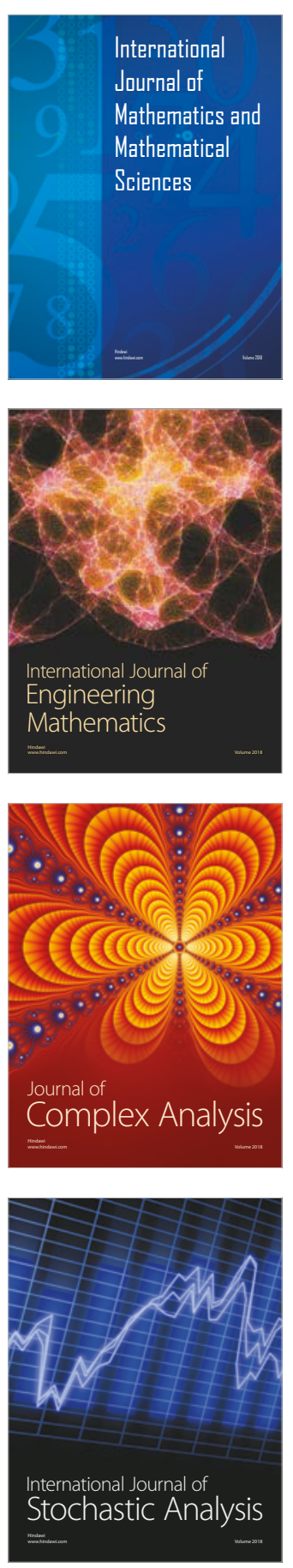
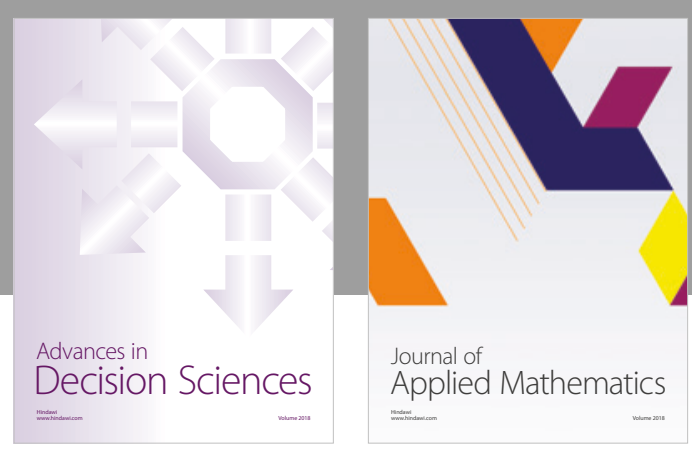

Journal of

Applied Mathematics
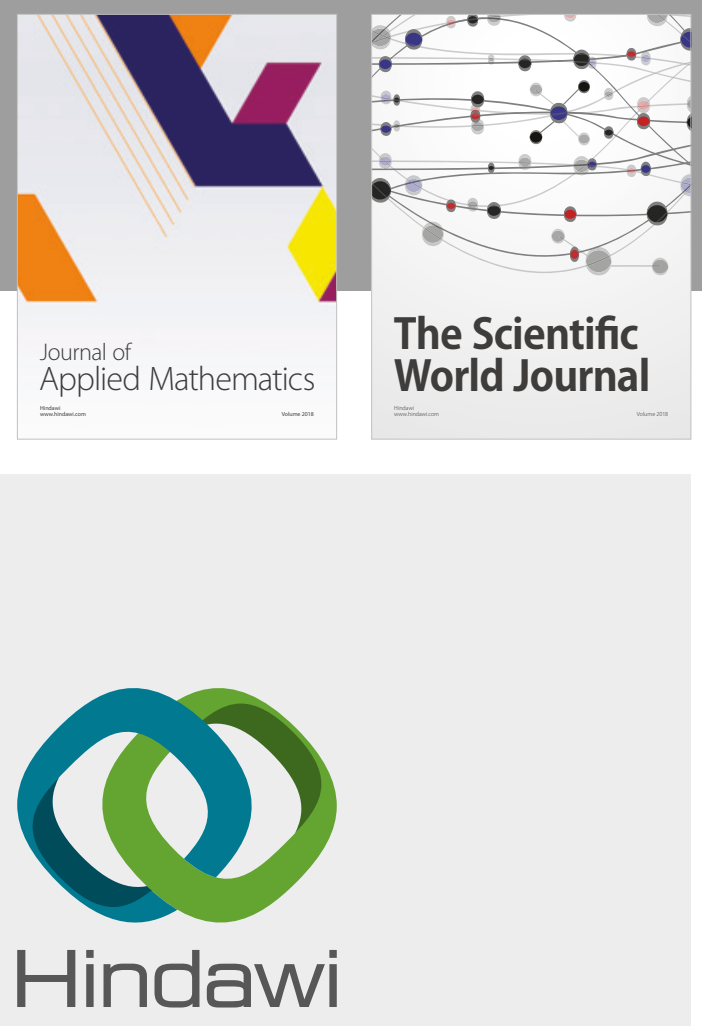

Submit your manuscripts at

www.hindawi.com

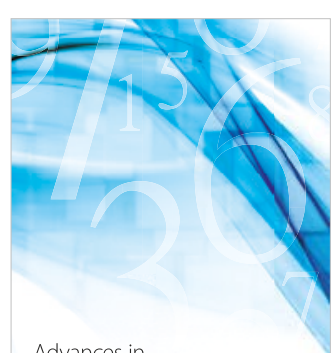

Advances in
Numerical Analysis
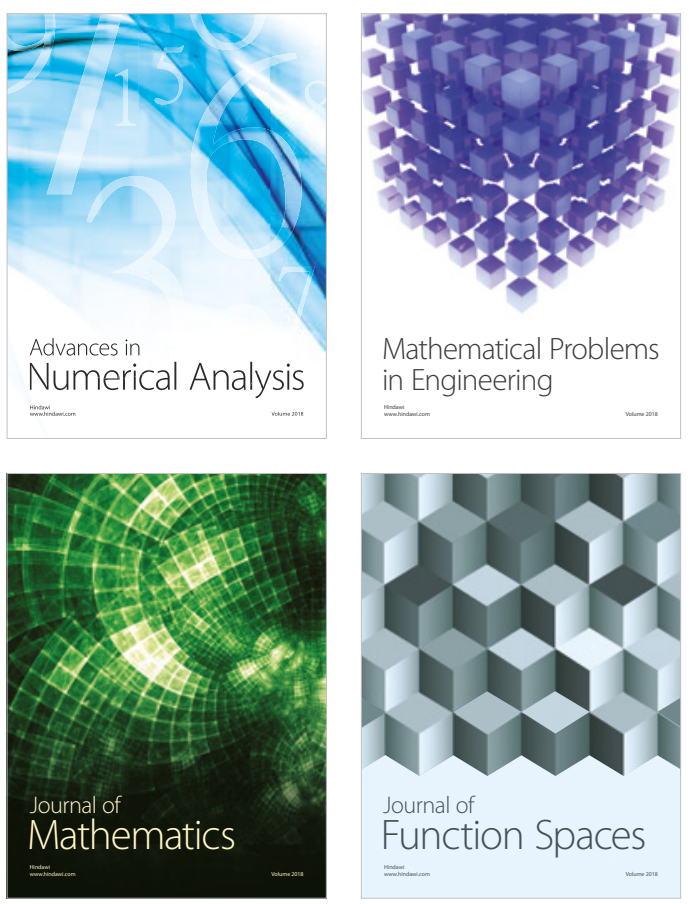

Mathematical Problems in Engineering

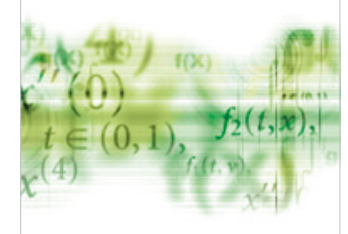

International Journal of

Differential Equations

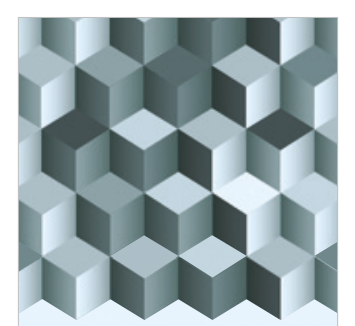

Journal of

Function Spaces

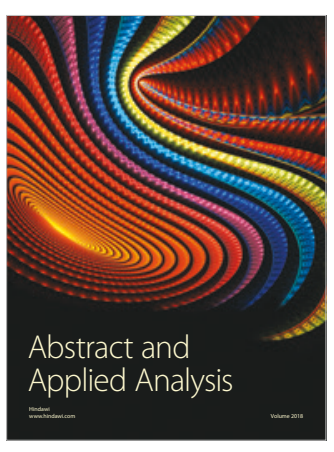

The Scientific

World Journal

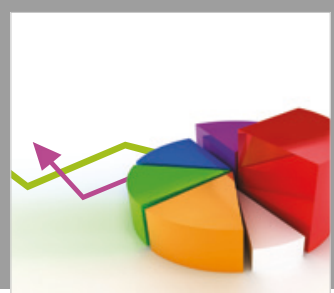

Journal of

Probability and Statistics
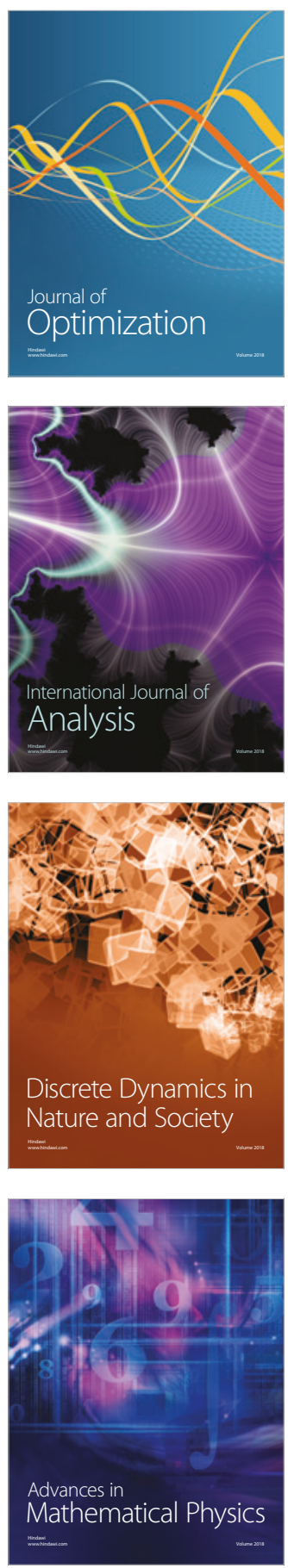